\title{
Global Sensitivity Analysis applied to Model Inversion Problems: A Contribution to Rail Condition Monitoring
}

\author{
René Schenkendorf ${ }^{1}$ and Jörn C. Groos ${ }^{1}$ \\ ${ }^{1}$ Institute of Transportation Systems, German Aerospace Center (DLR e.V.), Lilienthalplatz 7, 38108 Braunschweig, Germany \\ \{rene.schenkendorf,joern.groos\}@dlr.de
}

\begin{abstract}
Rising demands on railroad infrastructure operator by means of profitability and punctuality call for advanced concepts of Prognostics and Health Management. Condition based preventive maintenance aims at strengthening the rail mode of transport through an optimized scheduling of maintenance actions based on the actual and prognosticated infrastructure condition, respectively. When applying model-based algorithms within the framework of Prognostics and Health Management unknown model parameters have to be identified first. Which of these parameters should be known as precisely as possible can be figured out systematically by a sensitivity analysis. A comprehensive global sensitivity analysis, however, might be prohibitive by means of computation load when standard algorithms are implemented. In this study, it is shown how global parameter sensitivities can be calculated efficiently by combining Polynomial Chaos Expansion and Point Estimate Method principles. The proposed framework is demonstrated by a model inversion problem which aims to recalculate the track quality by measurements of the vehicle acceleration, i.e. analyzing the dynamic railway track-vehicle interaction.
\end{abstract}

\section{INTRODUCTION}

Railway networks are historically grown complex systems consisting of various types of infrastructures (sub)components. Nowadays, about 50 percent of the life cycle costs of railway infrastructures are made up by maintenance costs (Gradinariu et al., 2008). Here, the condition based preventive maintenance aims at cutting costs and increasing availability by an optimized scheduling of maintenance actions taking into account the actual infrastructure condition and its expected further degradation. Prerequisite therefore is the almost continuous condition monitoring for thousands of kilometers of railway tracks. One approach to account for this challenge is the development of low-cost railway track condition monitoring systems based on MEMS inertial sensors (accelerometers and

\footnotetext{
René Schenkendorf et al. This is an open-access article distributed under the terms of the Creative Commons Attribution 3.0 United States License, which permits unrestricted use, distribution, and reproduction in any medium, provided the original author and source are credited.
}

gyroscopes) which can be installed on usual in-line freight and passenger trains (e.g. (Ward et al., 2011; Molodova, Li, \& Dollevoet, 2011; Naganuma, Kobayashi, \& Tsunashima, 2013b)). In this article, mathematical models are utilized for the purpose of railway track condition monitoring by analyzing the dynamic railway track - vehicle interactions. More precisely, so-called inverse model problems are of special interest. An inverse model aims to recalculate previous inputs (irregularities) of the monitored system (rail surface) which had caused recorded system responses (dynamic vehicle reactions). These recalculated inputs are the basics of subsequent condition monitoring analyses. As in any model-based context uncertainties of the applied model parameters may influence the derived inputs significantly. Thus, a parameter sensitivity analysis might help to quantify the impact of parameter imperfections. In particular, it is shown how global parameter sensitivities can be calculated efficiently by combining Polynomial Chaos Expansion and Point Estimate Method principles. In so doing, a subset of model parameters can be identified which should be known as precisely as possibly to provide credible model-based results.

The remainder of this paper is organized as follows. In Section 2 an overview of rail condition monitoring concepts is given. In Section 3 a mechanistic model of the vehicle dynamic is derived. The basics of model inversion strategies are summarized in Section 4. Mathematical tools for parameter sensitivity analysis are introduced in Section 5. Sections 6 and 7 show how to apply the Polynomial Chaos Expansion and the Point Estimate Method for an efficient calculation of global parameter sensitivities. A numerical example given in Section 8 illustrates the proposed framework descriptively. Finally, the conclusion is given in Section 9

\section{Rail Condition Monitoring}

Of high importance for railway operators are rail infrastructure elements which reduce Reliability, Availability, Maintainability and Safety, so-called RAMS-killers, as well as drive up operating costs. A working group of the International Union of Railways (UIC) identified switches and crossings as most 
important RAMS-killers and the track geometry as well as rail head quality as the most important cost drivers (International Union of Railways (UIC), 2010). The recent approach to increase RAMS and to reduce costs is to establish optimized condition-based preventive maintenance strategies in the field of Prognostics ans Health Management (PHM). The task of a modern railway condition monitoring is therefore to provide a sufficient data base of reliable condition information. Once the current infrastructure condition can be reliably monitored and assessed, the future trend of infrastructure degradation is of utmost interest in condition based preventive maintenance. For instance, by applying suitable degradation models the remaining useful life (RUL) of technical devices (e.g. switch engines, signals) or critical track defects (e.g. track settlement/misalignment, rail fatigue) of rail segments under study can be prognosticated. Subsequently, ongoing maintenance actions can be re-scheduled optimally taking account of the actual infrastructure condition. Nowadays, rail condition monitoring is typically done in fixed time intervals by visual inspection, with manually operated measurement devices (hand-held, trolleys) or with measurement systems mounted on dedicated measurement trains. The recent developments to improve rail condition monitoring are to replace visual inspection by automatic procedures and to replace manually operated measurement systems by autonomous systems mounted on trains. These improvements reduce significantly the costs and track possession time for condition monitoring. At the same time, condition monitoring systems to be installed on in-line commercial trains are developed to further reduce costs and to provide a nearly continuous monitoring of railway track condition. Main foci for in-line condition monitoring systems are the two main costs drivers: defects of the rail head / rolling contact fatigue (e.g. head-checks, squats, corrugation (Fig. 1)) and misalignments of track geometry (vertical and lateral track alignment, gauge). One approach is the migration of (expensive) measurement systems from dedicated measurement trains to a small number of commercial in-line trains. This approach typically implies a significant shortening of the maintenance intervals for the equipped in-line vehicles. Another approach is the development of comparably low-cost railway track condition monitoring systems based on MEMS sensors (accelerometers, gyroscopes and microphones) which can be installed with low maintenance effort on usual in-line freight and passenger trains (e.g. (Ward et al., 2011; Lee, Choi, Kim, Park, \& Kim, 2012; Naganuma et al., 2013b; Molodova, Li, Nunez, \& Dollevoet, 2014; Mori, Sato, Ohno, Tsunashima, \& Saito, 2013; Bocciolone, Caprioli, Cigada, \& Collina, 2007; Weston, Ling, Roberts, et al., 2007)). These systems are based on the idea to utilize the dynamic train-trackinteraction caused by track defects for condition monitoring (e.g. (R. B. Lewis \& Richards, 1986; R. Lewis \& Richards, 1988)). The feasibility of this basic idea is proved by a number of numerical as well as experimental studies for defects of the rail (e.g. (Sunaga, Sano, \& Ide, 1997; Mori, Tsunashima, Ko- jima, Matsumoto, \& Mizuma, 2010; Molodova et al., 2011)) as well as track geometry (e.g. (Kawasaki \& Youcef-Toumi, 2002; Feldmann, Kreuzer, \& Pinto, 2000; M. Kobayashi, Naganuma, Nakagawa, \& Okumura, 2008; Weston, Ling, Goodman, et al., 2007; Weston, Ling, Roberts, et al., 2007)). The different approaches differ in the applied data analysis methodologies. The common approaches are based on model-free time domain, frequency domain or time-frequency analyses of the time domain acceleration data (e.g. (Caprioli, Cigada, \& Raveglia, 2007; Molodova et al., 2014; Feldmann et al., 2000; Naganuma, Kobayashi, \& Okumura, 2010)) as well as modelbased inversion methods (e.g. (T. Kobayashi, Naganuma, \& Tsunashima, 2013; Kawasaki \& Youcef-Toumi, 2002; Weston, Ling, Goodman, et al., 2007)).

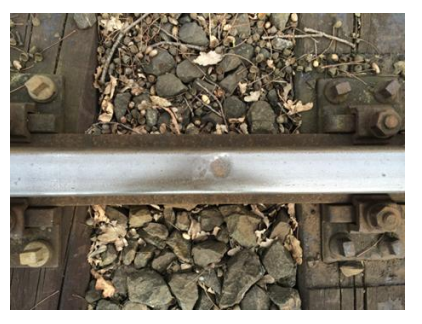

(a) Squat-type failure

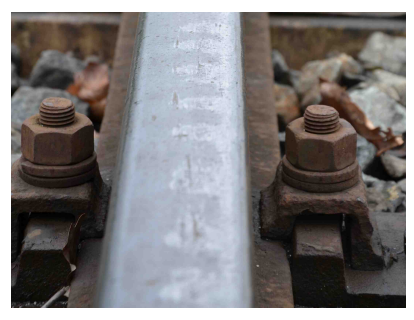

(b) Corrugation
Figure 1. Two snapshots of different rail surface failures.

The analyses by inversion methods are typically applied to accelerations measured at the bogie or the car body which are significantly influenced by the damping systems of the railway vehicles. Especially the utilization of car body accelerations is of high interest, as these can be measured with very low effort even by small portable measurement systems (e.g. (Mori et al., 2013)). Nevertheless, inversion analyses depend on a careful selection of parameters influencing the model outcome. Thus, a parameter sensitivity analysis should be mandatory in this context and deserves a detailed explanation. This paper is focused on the application of model-based analyses of the dynamic reactions of a rail vehicle to assess the condition of the rail surface (e.g. corrugation, squats). In particular, it is shown how global parameter sensitivities can be calculated efficiently by combining Polynomial Chaos Expansion and Point Estimate Method principles. Some illustrative, preliminary results and the conclusion complete this manuscript.

\section{Modeling of THE Vehicle Dynamics}

The dynamic response of the vehicle dynamic might be described by mechanical vehicle suspension systems. These systems may include the full vehicle-body motion up to single axis movements (Fig. 2) known as quarter-vehicle models (Imine, 2011; Naganuma, Kobayashi, \& Tsunashima, 2013a). For instance, the governing equations for a quarter-vehicle system are given as: 


$$
\begin{aligned}
m_{s} \ddot{x}_{s}= & -k_{s}\left(x_{s}-x_{u s}\right)-c\left(\dot{x}_{s}-\dot{x}_{u s}\right) \\
m_{u s} \ddot{x}_{u s}= & k_{s}\left(x_{s}-x_{u s}\right)+c\left(\dot{x}_{s}-\dot{x}_{u s}\right)+ \\
& k_{u s}\left(u(t)-x_{u s}\right)
\end{aligned}
$$

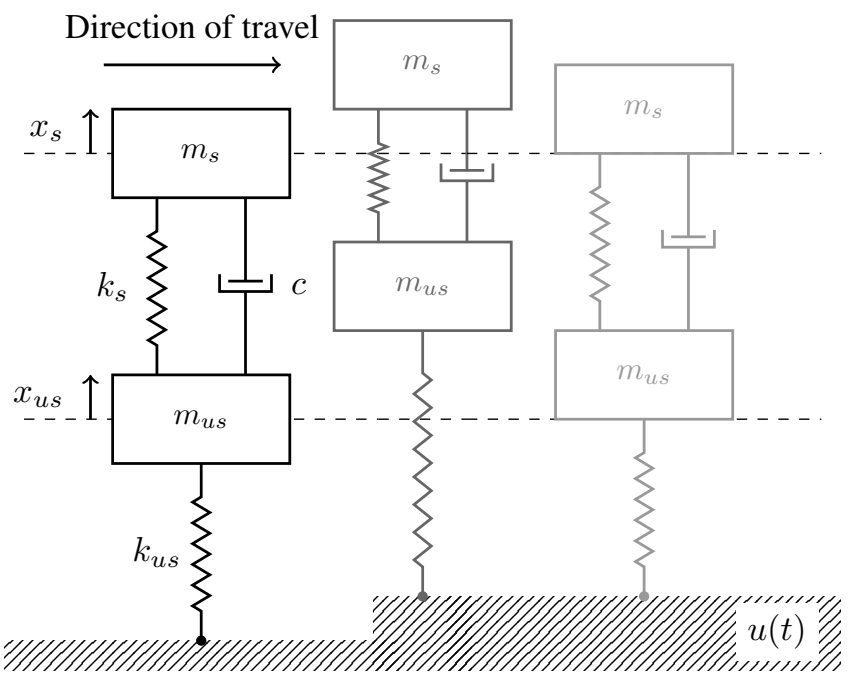

Figure 2. Mechanical suspension system representing the quarter-vehicle model. By this simplified model the dynamic railway track - vehicle interaction can be analyzed, e.g., the vehicle response when crossing track irregularities, $u(t)$.

Here, the differential equation system includes the sprung mass, $m_{s}$, and the unsprung mass of the vehicle, $m_{u s}$, which are connected by a linear spring and damper with the stiffness coefficient, $k_{s}$, and the damping constant, $c$, respectively. The rail surface is considered as the system input, $u(t)$, and is transmitted by a spring $\left(k_{u s}\right)$ to the unsprung mass. This kind of model, for example, might be applied to analysis vehicle dynamics in simulation studies as part of vehicle design phases. In Fig. 3 an example of the dynamic railway track - vehicle interaction is illustrated. A simulated track irregularity (Fig. 3a) causes accelerations of the sprung mass (Fig. $3 \mathrm{~b}$ ) and the unsprung mass (Fig. 3c), respectively. In this contribution, however, the reverse is of interest. Utilizing acceleration measurements to reconstruct track irregularities by model inversion concepts.

Table 1. Applied model parameters adapted from (SiraRamirez et al., 2011).

\begin{tabular}{|c|c|}
\hline Model parameter & Numerical value \\
\hline$m_{s}$ & $9875[\mathrm{~kg}]$ \\
$m_{u s}$ & $1100[\mathrm{~kg}]$ \\
$k_{s}$ & $2.13 \times 10^{6}[\mathrm{~N} / \mathrm{m}]$ \\
$k_{u s}$ & $1.42 \times 10^{8}[\mathrm{~N} / \mathrm{m}]$ \\
$c$ & $1.20 \times 10^{5}[\mathrm{~N}-\mathrm{s} / \mathrm{m}]$ \\
\hline
\end{tabular}

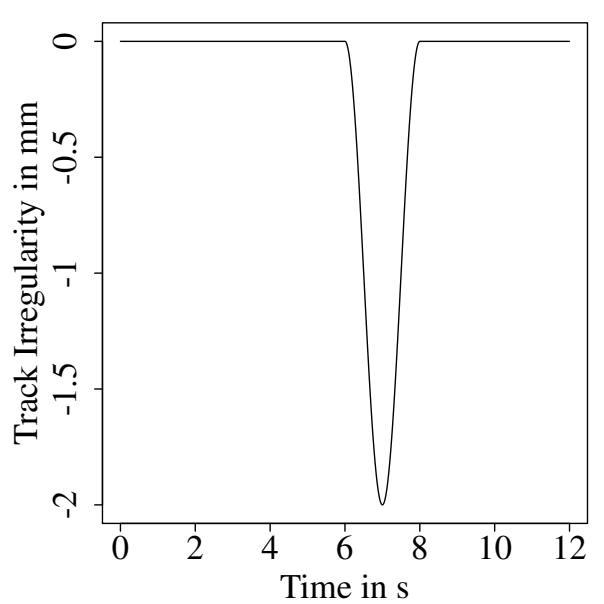

(a) Simulated track irregularity, $u(t)$.

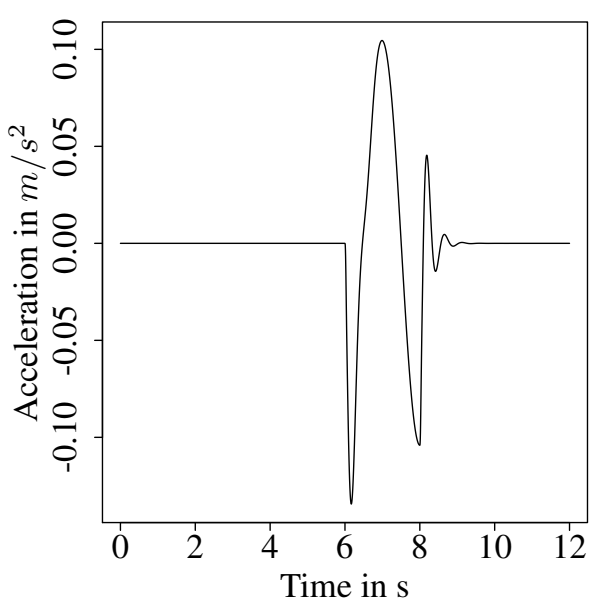

(b) Acceleration of the sprung mass, $m_{s}$.

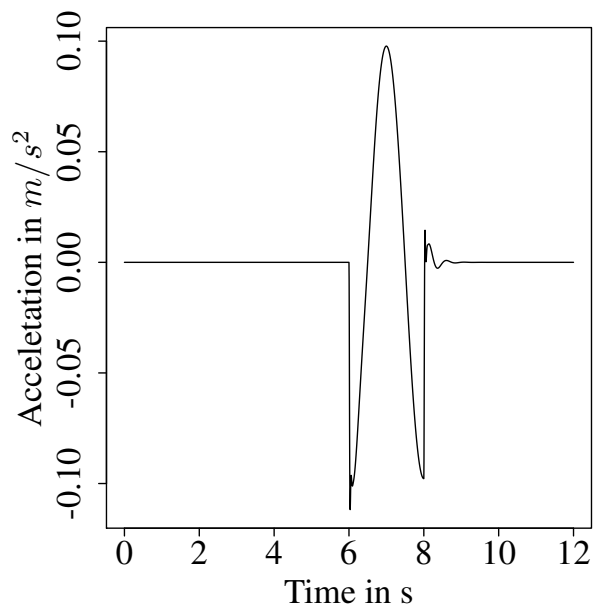

(c) Acceleration of the unsprung mass, $m_{u s}$.

Figure 3. Snapshot of the dynamic railway-vehicle interaction at a vehicle speed of $10 \mathrm{~m} / \mathrm{s}$. 


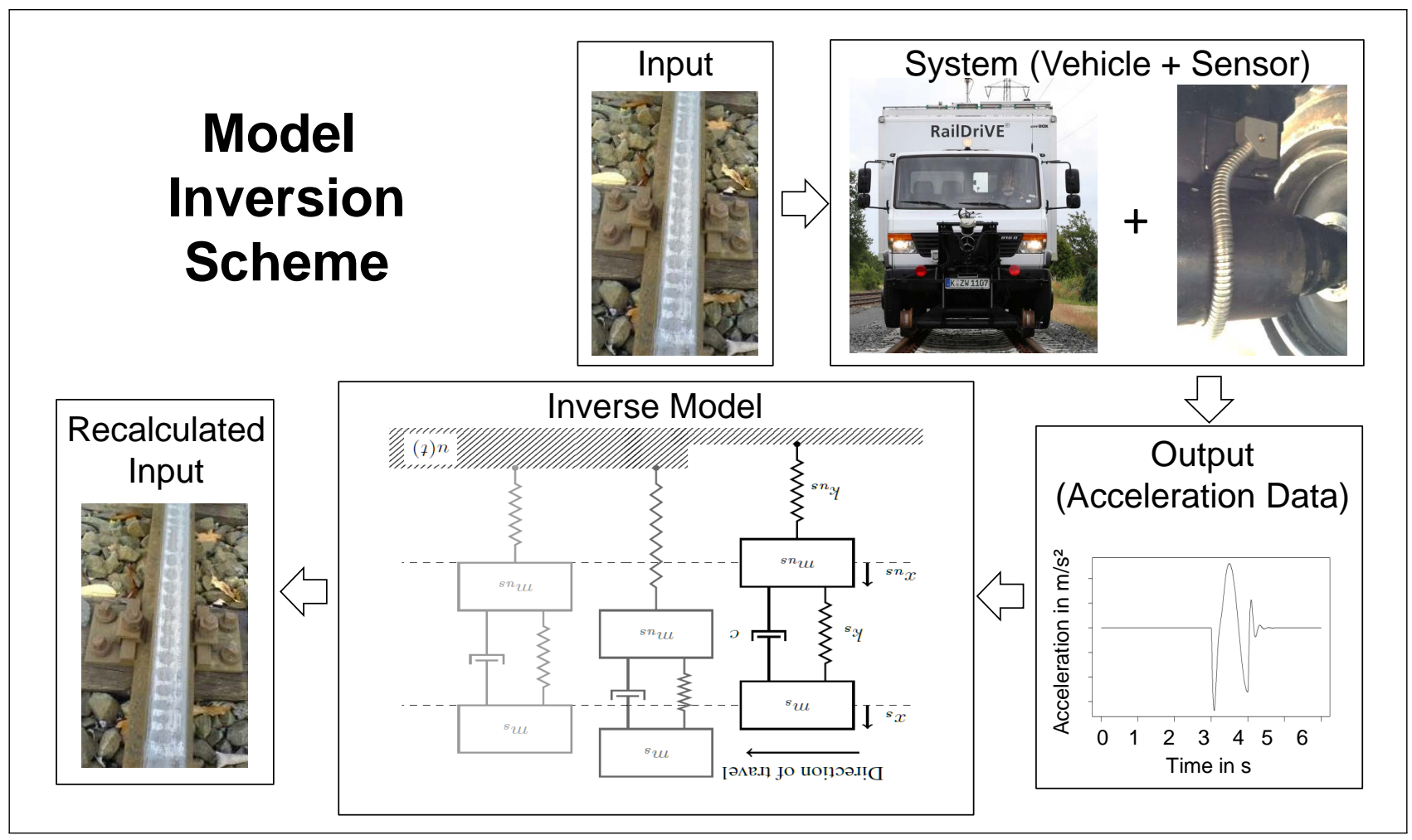

Figure 4. Workflow of Model Inversion: The DLR two-way vehicle RailDriVE is affected by the track quality. The resulting vehicle response given by acceleration data in combination with the inverse model is used to recalcualted the track quality.

\section{MOdel INVERSiON BY INVERSE Simulation}

The objective of model-based inverse solutions is to recalculate the time history of inputs which had caused a given model output result. These recalculated inputs might be applied to vehicles to follow a predefined trajectory (Thomson \& Bradley, 2006) or to force technical systems to desired operating conditions (Graichen, Hagenmeyer, \& Zeitz, 2005). In this contribution, however, the intention is a different one. Here, the focus is to recalculate model inputs which are associated to the rail track quality, i.e. rail surface failures (e.g. squats and corrugations) and rail track misalignments. As rail irregularities impact the mechanical train/vehicle suspension system, acceleration measurements of the resulting vehicle dynamic might be used to reconstruct the actual track quality by a model inversion strategy.

For the special case of Linear Time Invariant (LTI) systems the model can be represented in its state-space form:

$$
\begin{aligned}
& \dot{x}=A x+B u \\
& y=C x+D u
\end{aligned}
$$

where $u \in \mathcal{R}^{n_{u}}$ and $y \in \mathcal{R}^{n_{y}}$ are the systems inputs and the outputs, respectively. The system states are given by $x \in \mathcal{R}^{n_{x}}$. The system matrices are known as the dynamic matrix $A$, the input matrix $B$, the output matrix $C$, and the feedthrough matrix $D$.

Applied to the suspension system (Eq. (1)) the corresponding matrices assuming $\dot{x}=\left[\ddot{x}_{s}, \ddot{x}_{u s}, \dot{x}_{s}, \dot{x}_{u s}\right]^{\top}$ and $y=\ddot{x}_{s}$ are:

$$
\begin{aligned}
A & =\left[\begin{array}{cccc}
-\frac{c}{m_{s}} & \frac{c}{m_{s}} & -\frac{k_{s}}{m_{s}} & \frac{k_{s}}{m_{s}} \\
\frac{c}{m_{u s}} & -\frac{c}{m_{u s}} & \frac{k_{s}}{m_{u s}} & -\frac{\left(k_{s}+k_{u s}\right)}{m_{u s}} \\
1 & 0 & 0 & 0 \\
0 & 1 & 0 & 0
\end{array}\right] \\
B & =\left[\begin{array}{c}
0 \\
\frac{k_{u s}}{m_{u s}} \\
0 \\
0
\end{array}\right] \\
C & =\left[\begin{array}{llll}
-\frac{c}{m_{s}} & \frac{c}{m_{s}} & -\frac{k_{s}}{m_{s}} & \frac{k_{s}}{m_{s}}
\end{array}\right] \\
D & =[0]
\end{aligned}
$$

Within the inverse simulation concept the original model is extended by a feedback control loop (Murray-Smith, 2011). For the purpose of illustration the state-space model is transfered into the transfer function first, i.e. applying Laplace Transformation: 


$$
G(s)=C(s I-A)^{-1} B+D
$$

In general, the transfer function, $G(s)$, represents the input/output behavior of the system under study:

$$
G(s)=\frac{Y(s)}{U(s)}
$$

The corresponding closed-loop system (Fig. 5) reads as:

$$
G^{*}(s)=\frac{Y(s)}{U^{*}(s)}=\frac{K^{c}}{1+K^{c} \cdot G(s)}=\frac{K^{c} \cdot U(s)}{U(s)+K^{c} \cdot Y(s)}
$$

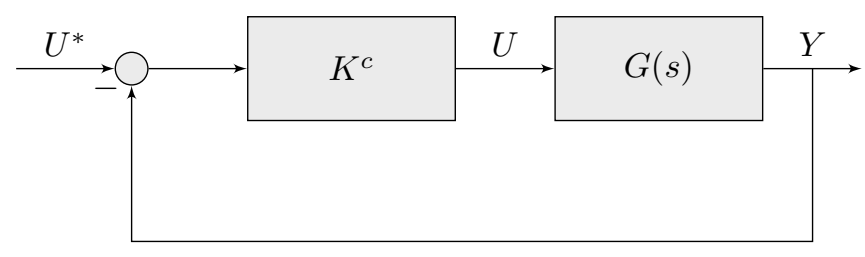

Figure 5. Control loop: Inverse simulation by a proportional feedback strategy.

In principle, for a large controller gain, $K^{c}$, the inverse system can be derived according to:

$$
\lim _{K^{c} \rightarrow \infty} G^{*}(s)=\frac{U(s)}{Y(s)}
$$

When Eq. (6) is transfered back into the state-space notation the corresponding LTI system reads as:

$$
\begin{aligned}
\dot{x}^{\dagger} & =A^{\dagger} x^{\dagger}+B^{\dagger} y \\
u & =C^{\dagger} x^{\dagger}+D^{\dagger} y
\end{aligned}
$$

where

$$
\begin{aligned}
A^{\dagger} & =\left(A-B K^{c} C\right) \\
B^{\dagger} & =B K^{c} \\
C^{\dagger} & =-K^{c} C \\
D^{\dagger} & =K^{c}
\end{aligned}
$$

At this point it should be stressed that the control gain has to be chosen deliberately to ensure a stable inversion (MurraySmith, 2011). Hence a stability analysis should be done in parallel when designing the control gain matrix, $K^{c}$.

In principle, alternative methods might be applied to the purpose of model inversion. As summarized in APPENDIX A, some of these methods have even been successfully applied to track monitoring issues and related problems. In general, to choose the best method for model inversion might be delicate. However, the universal problem of imprecise parameter estimates and their impact onto the simulation results applies to all model-based approaches universally. To reliably assess the contribution of parameter imperfections onto simulation results is still challenging and an important field of uncertainty analysis. To the best of the authors knowledge this is the first analysis of global sensitivities for inverse simulation models extending the local sensitivity framework (Murray-Smith, 2013).

\section{Sensitivity Analysis}

The large number of recent articles devoted to problems of uncertainty analysis/management in the field of PHM (e.g. (Saha, Goeble, Poll, \& Christophersen, 2009; Daigle \& Goebel, 2010; Daigle, Saxena, \& Goebel, 2012; Lapira, Brisset, Davari, Siegel, \& Lee, 2012; Williard, He, Osterman, \& Pecht, 2013; Sankararaman \& Goebel, 2013; Sankararaman, Daigle, Saxena, \& Goebel, 2013; Daigle \& Sankararaman, 2013; Kulkarni, Biswas, Celaya, \& Goebel, 2013; Zhang \& Pisu, 2014; Schenkendorf, 2014)) indicates the significance of this topic. Here, the parameter sensitivity analysis is an important aspect (e.g. (Sudret, 2007; Sankararaman, 2012; Chiachio et al., 2015)) because of the following reasons. The quality of any model-based result depends significantly on its applied model parameters. Typically, model parameters cannot be identified perfectly by measurements, i.e. measurement imperfections cause imprecise parameter estimates. Hence model parameters should be treated as random variables instead as deterministic quantities. Naturally, those parameters have to be identified as precisely as possible which impact the model response at most. In any model-building process one should be aware that some model parameters can be changed by order of magnitude without any detectable model response variation. Apart from that, slight displacements of other model parameters may have a severe impact, i.e. the model response is insensitive or sensitive to parameter variations/uncertainties. The insensitive group can be fixed at literature values (Sobol', Tarantola, Gatelli, Kucherenko, \& Mauntz, 2007), which may simplify the actual identification process of the sensitive parameters additionally as the measurement information is split to a reduced number of quantities.

A systematic quantification and classification of parameter impacts can be done by a sensitivity analysis. In this study essentials for the purpose of sensitivity analysis are briefly recapitulated. The general objective is to demonstrate how so-called global parameter sensitivities can be derived at low computational costs by implementing Polynomial Chaos Expansion (PCE) and Point Estimate Method (PEM) algorithms.

The dependency of model-based results to model parameters can be described generically by the following (non)-linear 
mapping problem:

$$
\eta=g(\theta)
$$

In particular, the simulation result, $\eta \in \mathcal{R}^{n_{\eta}}$, may express the vehicle dynamic (forward simulation; $\eta:=y$ ) and the rail quality (inverse simulation; $\eta:=u$ ), respectively. The model parameters (e.g. stiffness coefficients and damping constants) are given as elements of vector $\theta \in \mathcal{R}^{n_{\theta}}$. Depending on the test case, $g(\cdot)$ represents the classical feedforward model (e.g. Eq. (1)) and the inverse model (e.g. Eq. (7)), respectively. The impact of the ith model parameter onto the simulation result can be determined by a global sensitivity analysis.

In most global sensitivity analysis (GSA) methods the uncertainty of model parameters is addressed explicitly, i.e. modelbased results and parameters are considered as random variables. Most often as well as in this study, the uncertainty is represented by general second-order random variables (random variables of finite variance), i.e. $[\theta]_{i} \in L^{2}(\Omega, \mathcal{F}, \mathcal{P})$. The set $\Omega$ is a sample space, $\mathcal{F}$ is an appropriate $\sigma$-algebra on $\Omega$, and $\mathcal{P}$ is a probability measure (Grigoriu, 2002). Commonly, the global sensitivity is quantified by the analysis of variance (ANOVA principle). While operating in a pure probabilistic framework, the basic idea of so-called Sobol' Indices (SI-GSA) is to identify the variance contribution that each parameter, $[\theta]_{i}$, adds to the variance of the model-based result, $\eta$.

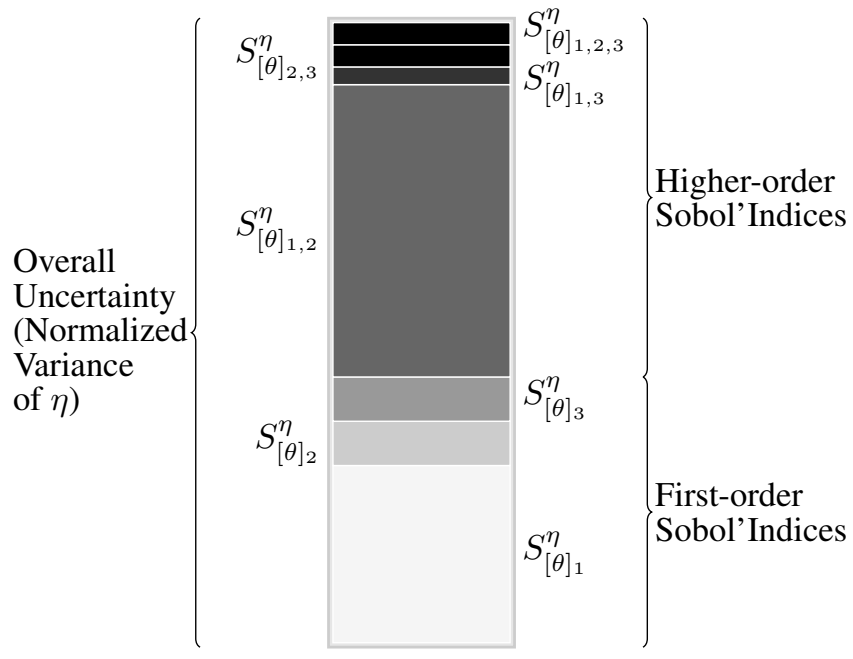

Figure 6. Sobol' Indices illustrated by a generic 3-dimensional parameter problem, $\theta \in \mathcal{R}^{3}$.

To address the joint impact of uncertain parameters (Fig. 6) higher-order Sobol' Indices can be derived additionally. For more details see (Saltelli, Ratto, Tarantola, \& Campolongo, 2005; Sobol', 1993) and references therein.

Obviously, SI-GSA takes the variance contribution into consideration solely. Hence effects caused by variations of the mean and higher moments (e.g. skewness or kurtosis) are neglected. Unsurprisingly, a parameter which cause a strong offset/bias onto the model-based result but contributes less to the response variance might be important for the model performance, too. Alternatively, so-called moment-independent importance measures have been recently introduced in the field of GSA (Borgonovo, 2007) analyzing the entire probability density function instead of isolated statistical moments.

The moment-independent global sensitivity analysis (MI-GSA) aims to identify the impact of a parameter, $[\theta]_{i}$, onto the modelbased result and its probability density distribution (PDF), respectively (Fig. 7). Explicitly, the difference between the unconditional PDF, $p d f(\eta)$, and a conditional PDF, $p d f\left(\eta \mid[\theta]_{i}\right)$, is determined according to:

$$
s\left([\theta]_{i}\right)=\int_{\Omega}\left|p d f(\eta)-p d f\left(\eta \mid[\theta]_{i}\right)\right| d \eta
$$

To address the uncertainty of $[\theta]_{i}$ the expected value of $s\left([\theta]_{i}\right)$ has to be analyzed:

$$
E\left[s\left([\theta]_{i}\right)\right]=\int_{\Omega} p d f\left([\theta]_{i}\right) s\left([\theta]_{i}\right) d[\theta]_{i}
$$

Finally, the normalized importance measure of MI-GSA (Borgonovo, 2007) is given by:

$$
\delta_{i}=\frac{1}{2} E\left[s\left([\theta]_{i}\right)\right]
$$

Similar to the Sobol' Indices, an insensitive factor has an importance measure close to zero and the overall sum holds the inequality:

$$
\sum_{i=1}^{n_{\theta}} \delta_{i} \leq 1
$$

To sum up MI-GSA is an appropriate concept for the purpose of sensitivity analysis, its numerical calculation, however, might be a challenge. In most cases, the required probability density functions cannot be calculated analytically and have to be derived numerically, e.g. by Kernel density approximations. This is achieved by a large number of model runs representing the variation of the uncertain parameters. In case of cpuintensive model evaluations this might be prohibitive due to limited cpu-power and time, respectively. A remedy might be to evaluate handy surrogate functions, $\hat{g}(\cdot)$, instead. Here, the Polynomial Chaos Expansion comes into play and is applied to bypass potential cpu-intensive processes as indicated in Fig. 8. The basics of PCE and its efficient parameterization by the Point Estimate Method are described in the sequel. 


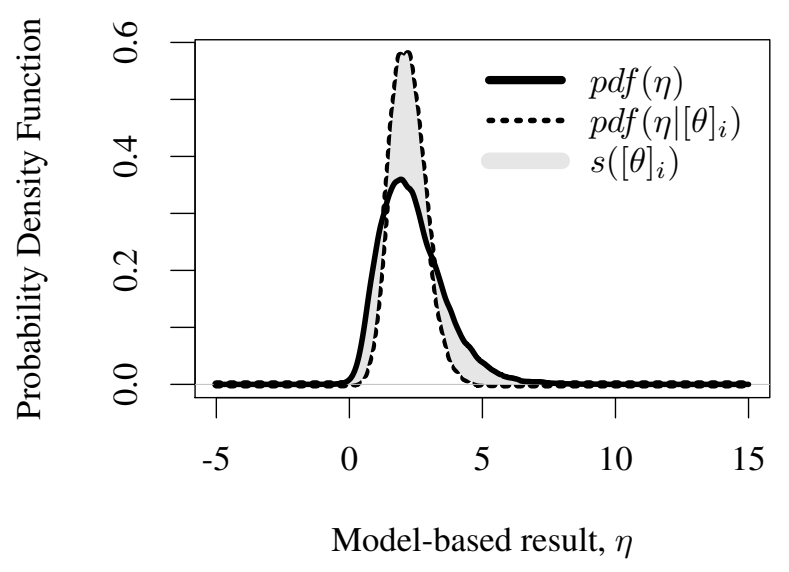

Figure 7. Difference, $s\left([\theta]_{i}\right)$, between the unconditional PDF, $p d f(\eta)$, and the conditional PDF, $p d f\left(\eta \mid[\theta]_{i}\right)$, is highlighted.

\section{Polynomial Chaos Expansion}

To ensure a computationally tractable calculation, Polynomial Chaos Expansion (PCE) is used to propagate the probabilistic parameter uncertainties onto the model-based results. In detail, PCE aims to represent the model response by a weighted superposition of deliberately chosen basis functions, i.e. orthogonal polynomials (Maitre \& Knio, 2010), $\Psi_{i}(\cdot)$, according to:

$$
\eta=g(\xi)=\sum_{i=0}^{\infty} a_{i} \Psi_{i}(\xi)
$$

where $[\xi]_{i} \sim \mathcal{N}(0,1)$, i.e. the elements of $\xi$ follow a standard Gaussian distribution and are uncorrelated to each other.

For practical applications the expansion is implemented in truncated form $\left(l_{p c e}<<\infty\right)$ as:

$$
\eta \approx \hat{\eta}:=\sum_{i=0}^{l_{p c e}} a_{i} \Psi_{i}(\xi)=a^{\top} \Lambda(\xi)
$$

where $a:=\left[a_{0}, \ldots, a_{l_{p c e}}\right]^{\top}$ and $\Lambda:=\left[\Psi_{0}, \ldots, \Psi_{l_{p c e}}\right]^{\top}$.

The model response is characterized by the PCE coefficients, $a_{i}$, and can be derived via:

$$
a_{i}=\frac{\int_{\Omega} g(\xi) \Psi_{i} p d f(\xi) d \xi}{\int_{\Omega} \Psi_{i}(\xi)^{2} p d f(\xi) d \xi}
$$

The denominator may be solved analytically (Maitre \& Knio, 2010), the numerator of Eq. (15), however, has to be calculated numerically. To keep the computational load tractable the integrals have to be evaluated by efficient sampling strategies. Commonly, standard Monte Carlo simulations and Quadrature methods involve an infeasible sample number, i.e. their

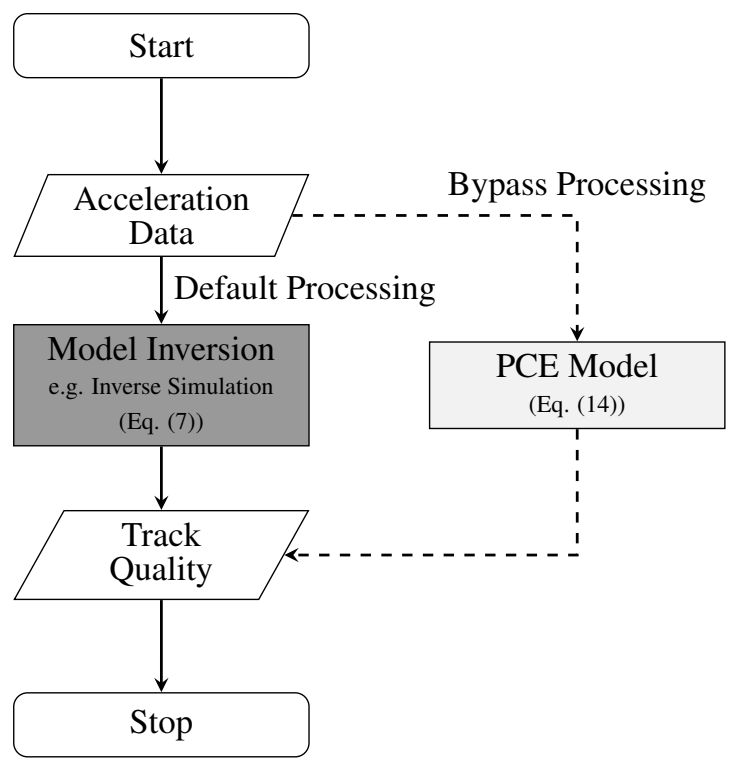

Figure 8. The cpu-intensive process of model inversion (dark gray) is bypassed by a fast to evaluate PCE surrogate model (light gray).

application is prohibitive in most cases. In this study, the Point Estimate Method (PEM) is implement as a practicable alternative. As explained in Sec. 7, by combining PCE with PEM the overall required sample number scales polynomially (quadratic or cubic) with the dimension of $\xi$ (the number of random elements / parameters). Obviously, this corresponds to a significant reduction in comparison to standard numerical integration methods, e.g. Gaussian Quadrature (exponential growth) or Monte Carlo simulations (educated guess: sample number $\geq 10,000$; and challenging to quantify in general) (Maitre \& Knio, 2010). Once parameterized (Eq. (15)), the surrogate model response, $\hat{\eta}$, can be derived at relative low computational costs as the cpu-intensive process of model inversion is bypassed by PCE (Fig. 8). Only now, Monte Carlo simulations propagating the uncertain parameters onto the model response become feasible. Subsequently, associated PDFs can be derived from these accelerated Monte Carlo simulations by applying a Gaussian Kernel density approximation (Bishop, 2008) as:

$$
p d f(\eta)=\frac{1}{N h} \sum_{i=1}^{N} K\left(\frac{\eta-\hat{\eta}_{i}}{h}\right)
$$

with the bandwidth parameter $h$, the sample number $N$, and the Gaussian Kernel function:

$$
K\left(\frac{\eta-\hat{\eta}_{i}}{h}\right)=K(z):=\frac{1}{\sqrt{2 \pi}} e^{-\frac{1}{2} z^{2}}
$$

So far, only standard Gaussian random variables, $[\xi]_{i}$, have 
been assumed. A way to incorporate general Gaussian random variables, which may describe problem dependent parameter uncertainties $\left([\theta]_{i} \sim \mathcal{N}\left(\mu_{i}, \sigma_{i}\right)\right)$, reads as:

$$
[\theta]_{i}:=q_{i}\left([\xi]_{i}\right)=\mu_{i}+\sigma_{i}[\xi]_{i}
$$

Similar transformation functions, $q_{i}(\cdot)$, can be derived for different types of parametric PDFs, too, as summarized in Tab. 2 .

Table 2. Probability density function transformation formulas adapted from (Isukapalli, 1999). Here, the term erf means the error function.

\begin{tabular}{|c|c|}
\hline Type of PDF & Transformation: $q_{i}\left([\xi]_{i}\right):=$ \\
\hline Normal $(\mu, \sigma)$ & $\mu+\sigma[\xi]_{i}$ \\
Uniform $(a, b)$ & $a+(b-a)\left(\frac{1}{2}+\frac{1}{2} \operatorname{erf}\left([\xi]_{i} \sqrt{2}\right)\right)$ \\
$\log -\operatorname{normal}(\mu, \sigma)$ & $\exp \left(\mu+\sigma[\xi]_{i}\right)$ \\
$\operatorname{Gamma}(a, b)$ & $a b\left([\xi]_{i} \sqrt{\frac{1}{9 a}}+1-\frac{1}{9 a}\right)^{3}$ \\
$\operatorname{Exponential}(\lambda)$ & $-\frac{1}{\lambda} \log \left(\frac{1}{2}+\frac{1}{2} \operatorname{erf}\left(\frac{[\xi]_{i}}{\sqrt{2}}\right)\right)$ \\
\hline
\end{tabular}

Such an isoprobabilisitic transform (Sudret, 2007) can also be applied to incorporate non-parametric empirical PDFs (Schöniger, Nowak, \& Franssen, 2012) as well. Thus an efficient sampling strategy for (standard) Gaussian distributions is of utmost significance within the proposed framework of sensitivity analysis. For this purpose the Point Estimate Method is tailor-made and explained in the sequel.

\section{Point Estimate Method}

In the last two decades the so-called Unscented Transformation (UT) introduced by Julier and Uhlmann in 1994 (Julier \& Uhlmann, 1994) has become the gold standard in non-linear filtering. The roots of UT, however, date back more than 60 years in time (Tyler, 1953). In detail, Point Estimate Methods (PEM) had been introduced to solve multi-dimensional integration problems over symmetrical regions, e.g. symmetric probability density functions (Evans, 1967, 1974). Due to this symmetry, numerical integration techniques can be derived which scale polynomially (quadratic or cubic) to ndimensional integration problems (e.g. Eq. (15)). Essentials of PEM are briefly reviewed in the sequel following annotations given in (Tyler, 1953; Lerner, 2002).

The main idea of PEM as a sampling approach is to generate sample points, $\xi_{k}$, by permuting at most one element of $\xi \in$ $\mathcal{R}^{n_{\xi}}$ at once as:

$$
\left[\xi_{k}\right]_{i}:=\left[\xi_{0}\right]_{i} \pm \vartheta
$$

Due to combinatorics $2 n_{\xi}$ sample points can be derived additionally to the original random vector, $\xi_{0}=E[\xi]$. To determine the permutation constant, $\vartheta$, and the associated sampling weights, $w_{i}$, the first raw statistical moments of $\xi$ are utilized (Lerner, 2002) and read as:

$$
\begin{aligned}
w_{0} & =1-\frac{n_{\xi}}{\vartheta^{2}} \\
w_{1} & =\frac{1}{2 \vartheta^{2}} \\
\vartheta & =\sqrt{3}
\end{aligned}
$$

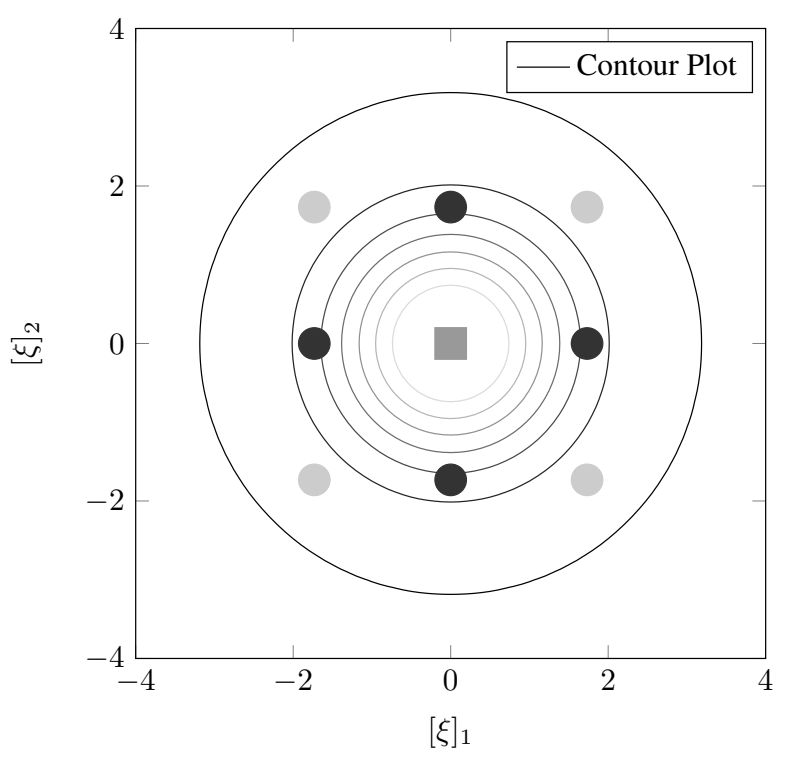

Figure 9. Bivariate standard Gaussian distribution: Contour plot superimposed by sample points generated by PEM3( $(, 0)$ and PEM5( $(\bullet, \odot)$, respectively.

Now, an $n_{\xi}$-dimensional integration problem can be solved approximatively by a weighted superposition of function evaluations at these deliberately chosen sample points according to:

$$
\int_{\Omega} g(\xi) p d f_{\xi} d \xi \approx w_{0} g\left(\xi_{0}\right)+w_{1} \sum_{k=1}^{2 n_{\xi}} g\left(\xi_{k}\right)
$$

As only a finite number of raw moments of the input random variable, $\xi$, is considered, a non-linear function, $g(\cdot)$, is approximated by monomials of finite degree (Evans, 1967; Lerner, 2002). For the proposed sampling strategy (Eqs. (19)-(20)) monomials up to order three are approximated correctly and consequently labeled as PEM3 in what follows.

Moreover, the general precision of the PEM approach can be increased gradually by incorporating an increased sample number and considering higher order raw moments of $\xi$, respectively. Hence an approximation scheme can be applied which represents monomials of $g(\cdot)$ correctly up to the preci- 
sion of 5 via:

$$
\begin{array}{r}
\int_{\Omega} g(\xi) p d f_{\xi} d \xi \approx w_{0} g\left(\xi_{0}\right)+ \\
w_{1} \sum_{k=1}^{2 n_{\xi}} g\left(\xi_{k}\right)+w_{2} \sum_{f=1}^{2 n_{\xi}\left(n_{\xi}-1\right)} g\left(\xi_{f}\right)
\end{array}
$$

The approximation scheme given in Eq. (21) is labeled as PEM5 subsequently. In this case, the number of generated sample points correlates to $2 n_{\xi}^{2}+1$ for a $n_{\xi}$-dimensional integration problem. For the purpose of parametrization of $w_{i}$ and $\vartheta$ an equation system can be derived taking into account monomials of degree 5 or less (Lerner, 2002).

The four unknowns can be uniquely determined as:

$$
\begin{aligned}
\vartheta & =\sqrt{3} \\
w_{0} & =1+\frac{n_{\xi}^{2}-7 n_{\xi}}{18} \\
w_{1} & =\frac{4-n_{\xi}}{18} \\
w_{2} & =\frac{1}{36}
\end{aligned}
$$

Resulting sample points, for example, are illustrated in Fig. (9) for a bivariate standard Gaussian distribution.

In summary, the proposed PEM5 framework provides a tradeoff between accuracy and computational demands and is applied in subsequent considerations for this very reason. The deliberately chosen sample points can be applied to determine the PCE coefficients (Eq. (15)) efficiently. In so doing, Sobol' Indices might be derived immediately by the PCE coefficients (Sudret, 2007; Alexanderian, 2013) at a total cost of $2 n_{\xi}^{2}+1$ function evaluations. As conditional probabilities have to be incorporated in case of MI-GSA explicitly, the overall sample number scales cubically (while eliminating redundant identical samples):

$$
\text { Sample Number }(P E M 5)=2 n_{\xi}^{3}-6 n_{\xi}^{2}+10 n_{\xi}-3
$$

Thus, the added value of MI-GSA has to be paid for by an increased (but manageable) computational demand. An effort which is worth to take into consideration in many practical studies of sensitivity analysis. The numerical results given in Sec. 8 confirm the usefulness of the proposed concept convincingly.

\section{Numerical Results}

First, the general applicability of acceleration data to track irregularity detection is assessed. When comparing the dynamic vehicle response (Eq. (3)) by means of the frequency response, Bode Diagram (Fig. 10), it can be seen that: i) Excluding stationary changes (very low frequencies) both sensor setups, i.e. acceleration data of the unsprung mass and the sprung mass, respectively, are feasible configurations. ii) Track irregularities impact the unsprung mass at most, i.e. associated acceleration data are more informative. Thus data of the unsprung mass might be preferable in theory.

In this study, however, the focus is on acceleration data of the sprung mass for the simple reason that the sensor hardware can be installed inside of the vehicle body and maintained easily a basic requirement for the intended purpose of a continuously operating rail condition monitoring system on a large number of commercial in-line trains.

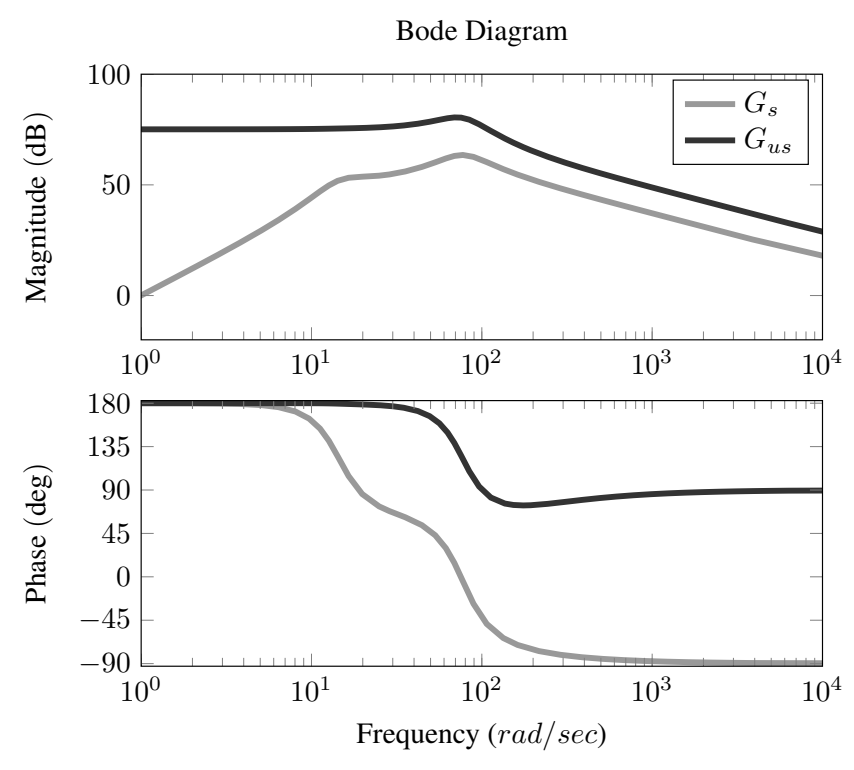

Figure 10. Bode Diagram of the proposed quarter-vehicle model assuming the acceleration of the sprung mass $\left(G_{s}\right)$ and the unsprung mass $\left(G_{u s}\right)$ as the system output, respectively.

\subsection{Scenario 1: Inverse Simulation}

In this scenario simulated acceleration data of the sprung mass $m_{s}$ are utilized to recalculate the rail track quality. To do so, the quarter-vehicle model (Eq. (1)) has to be inverted. As the input (rail surface) is indirectly linked to the sprung mass, an analytical inversion (Sec. 4) fails. When measuring the sprung mass acceleration, the system output, $y^{\operatorname{sim}}(t)=d^{2} x_{s} / d t^{2}$, is not a flat output $\left(y^{f}(t) \neq y^{\text {sim }}(t)\right.$ ). In consequence, also the flatness approach (Sec. A.2) is not directly applicable. As measurement imperfections are neglected, the Inverse Simulation (Sec. 4) is favored over Kalman filtering (Sec. A.3) for simplicity and implemented for subsequent analysis.

Assuming a perfect model (model imperfections will be ex- 
plicitly addressed in ongoing work) as well as perfect model parameters rail irregularities can be reconstructed perfectly by the inverse simulations approach (Fig. 11). Here, a control gain of $K^{c}=1000$ is applied.

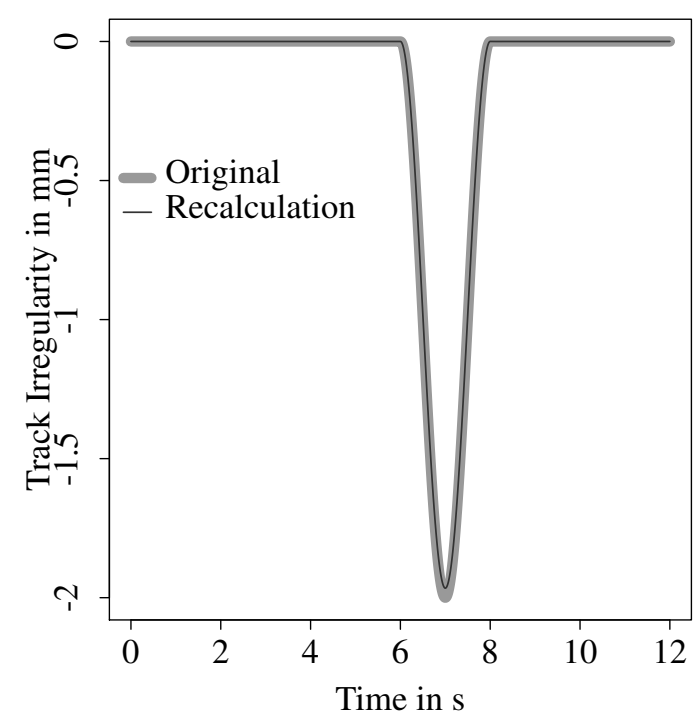

Figure 11. Benchmark of the original and the recalculated track irregularity: Almost no differences can be seen.

To be more realistic, it is assumed that the stiffness constants and the damping constant are only vaguely known, i.e. these 3 model parameters are described by random variables (Tab. 3).

Table 3. Assumed parameter uncertainties following a Gaussian distribution, $[\theta]_{i} \sim \mathcal{N}\left(\mu_{i}, \sigma_{i}\right)$.

\begin{tabular}{|c|c|}
\hline Mean Value & Standard Deviation \\
\hline$\mu_{k_{s}}=k_{s}$ & $\sigma_{k_{s}}=1 / 3 k_{s}$ \\
$\mu_{k_{u s}}=k_{u s}$ & $\sigma_{k_{u s}}=1 / 3 k_{u s}$ \\
$\mu_{c}=c$ & $\sigma_{c}=1 / 3 c$ \\
\hline
\end{tabular}

In this case, the uncertainty of the model parameters is transfered onto the model-based results. For instance, in Fig. 12 a histogram of the recalculated track quality is illustrated at the time point of maximum displacement $(t=7 s)$ shown in Fig. 11. This histogram is based on 100.000 accelerated Monte Carlo simulations, i.e. a truncated third-order PCE model (Eq. (14)) is evaluated instead of the inverse model (Eq. (7)). Here, the parameterization of the PCE model was done by 9 $\left(2 n_{\xi}^{2}+1 ; n_{\xi}=3\right)$ evaluations of the original inverse model which means a significant reduction in computational costs (i.e. 100.000 runs of the cpu-intensive inverse model). In principle, advanced sampling methods like Importance Sampling and MCMC can be applied to the surrogate model as well to improve the overall computational time additionally.

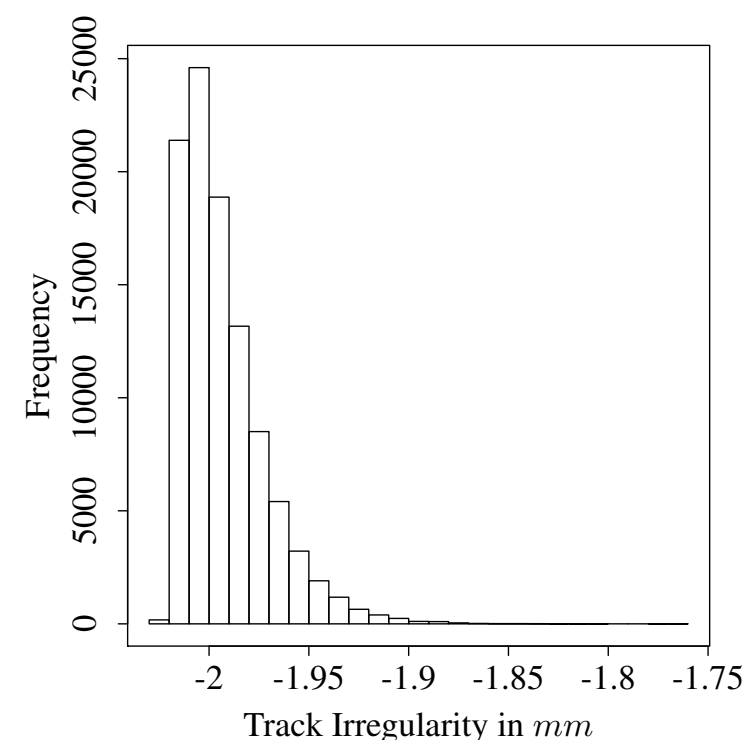

Figure 12. Histogram of the recalculated track irregularity $(t=$ $7 s$ ) representing the uncertainty induced by model parameter imperfections.

In a similar way, the associated model parameter sensitivities (MI-GSA) are derived at a total cost of 27 (Eq. (22)) cpuintensive inverse simulations (Eq. (7)). Any subsequent calculation is based on easily to evaluate PCE model surrogates (Eq. (14)). For instance, parameter sensitivities associated to the recalculated track quality are illustrated in Fig. 13 for time point $t=7 \mathrm{~s}$.

Obviously, the stiffness constant $k_{s}$ has the strongest impact onto the recalculated track quality at $t=7 \mathrm{~s}$. Minor contributions of the parameters $k_{u s}, c$, and parameter combinations $\left\{k_{s}, k_{u s}, c\right\}$ can also be detected. In consequence, the model parameter $k_{s}$ has to be known as precisely as possible to ensure a reliable recalculation of track irregularity maximum.

\subsection{Scenario 2: Classical Simulation}

The proposed concept of an efficient global parameter sensitivity analysis can also be applied to classical simulations, e.g. to simulate the vehicle response (Eq. (1)) for given artificial track quality configurations. Here, the same parameter uncertainties as in the previous study and the same track profile as shown in Fig. 11 are assumed. In Fig. 14 parameter sensitivities are shown of the simulated sprung mass acceleration at time point $t=7 \mathrm{~s}$. Compared to the sensitivities derived for the inverse simulation, a different range of parameter sensitivities can be seen. Here, the stiffness parameter $k_{s}$ determines the simulation result almost exclusively. In principle, it may happen that a sensitive model parameter for the classical simulation is insensitive for the inverse simulation and vice versa. Thus individual sensitivity analysis studies for the inverse simulation 


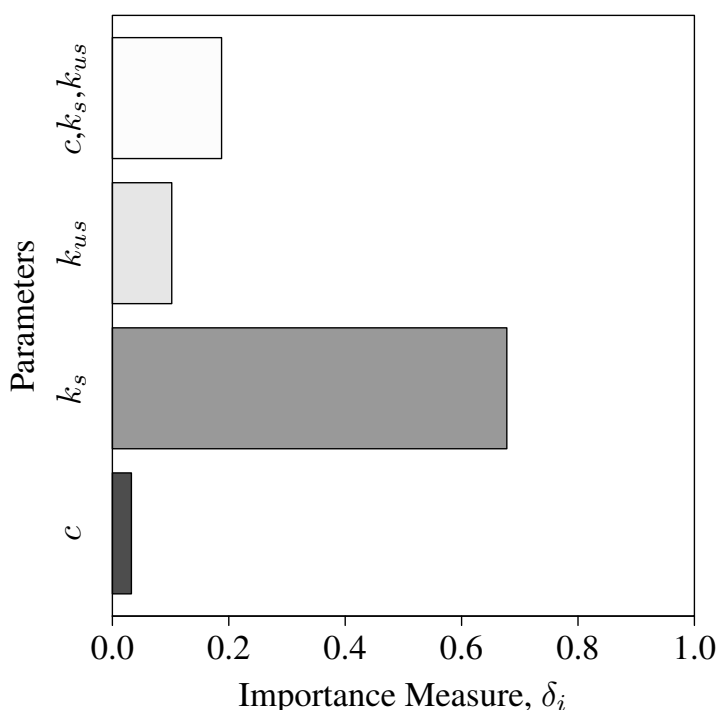

Figure 13. Sensitivity Analysis applied to the inverse simulation.

have to be performed.

\section{Conclusion}

In this study it was shown how global parameter sensitivities can be derived efficiently by combining Polynomial Chaos Expansion and Point Estimate Method principles. Considerations which are relevant for any model-based analyses, thus for model-based concepts in PHM, too. In this regard, the general idea of a model-based rail condition monitoring setup was introduced. A model inversion strategy enabled the recalculation of the track quality by incorporating acceleration data of the vehicle dynamic. Which model parameters have to be determined as precisely as possible to ensure meaningful calculations could be identified efficiently by the proposed approximation and sampling scheme. It is common knowledge that a comprehensive sensitivity analysis contributes to model calibration and refinement. Improved models in PHM ensure more credible model-based prognostic results in consequence. In future, sensitivity studies of more complex inverse models, e.g. full-vehicle models, will be analyzed.

\section{ACKNOWLEDGMENT}

We thank apl. Prof. Dr.-Ing. Michael Mangold for his helpful comments on a previous version of the paper. Moreover, the anonymous reviewers deserve special thanks for their valuable input. The financial support by Deutsches Zentrum fur Luftund Raumfahrt (DLR e.V., Cologne) under grant TrackScan is gratefully acknowledged.

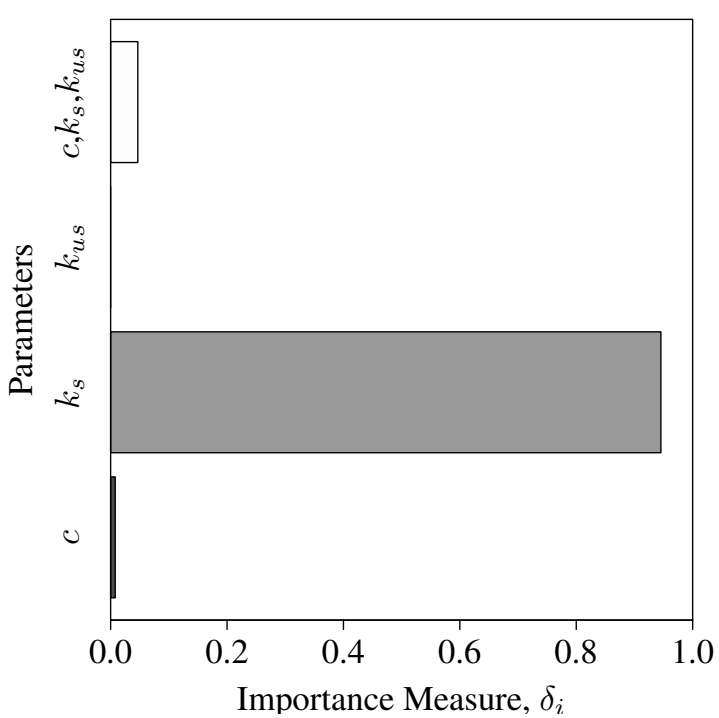

Figure 14. Sensitivity Analysis applied to the classical simulation.

\section{A. ApPEndix}

Alternative mathematical tools which can be applied to model inversion are shortly summarized below following notations given in (Buchholz \& v. Grünhagen, 2007; Murray-Smith, 2011).

\section{A.1. Analytical Inversion}

Assuming a regular $D$ matrix the input can be determined by given outputs as:

$$
u=D^{-1}(y-C x)=-D^{-1} C x+D^{-1} y
$$

Inserted in Eq. (2) the corresponding LTI reads as:

$$
\begin{aligned}
\dot{x}^{*} & =A^{*} x^{*}+B^{*} y \\
u & =C^{*} x^{*}+D^{*} y
\end{aligned}
$$

where

$$
\begin{aligned}
A^{*} & =\left(A-B D^{-1} C\right) \\
B^{*} & =B D^{-1} \\
C^{*} & =-D^{-1} C \\
D^{*} & =D^{-1}
\end{aligned}
$$

In the common case of non-regular $D$ matrices, however, alternative methods (e.g. inverse simulation) have to be applied.

\section{A.2. Flatness Approach}

The concept of differential flatness (Fliess, Lévine, Martin, \& Rouchon, 1992, 1995) is applicable for linear as well as non- 
linear systems and aims to represent the system states and the inputs by so-called flat outputs, $y^{f}(t)$, and derivatives thereof. The determination of these flat outputs might be challenging. In most cases, flat outputs do not agree with originally given output configurations, $y^{f}(t) \neq y^{\text {sim }}(t)$, but have to be defined appropriately. If a flat output exists the states and inputs can be expressed as:

$$
\begin{aligned}
& x(t)=\Psi_{x}\left(y^{f}(t), \mathcal{D} y^{f}(t), \ldots, \mathcal{D}^{n-1} y^{f}(t)\right) \\
& u(t)=\Psi_{u}\left(y^{f}(t), \mathcal{D} y^{f}(t), \ldots, \mathcal{D}^{n} y^{f}(t)\right),
\end{aligned}
$$

where $\mathcal{D}^{i}$ represents the operator notation of the $i$ th derivative, $d^{i} / d t^{i}$.

Recently, the flatness approach has been applied to an active regulation concept of a railway vehicle suspension system (Sira-Ramirez et al., 2011). In general, however, a successful application depends on the relative degree of the system under study. In case of single input single output LTI systems this corresponds to the difference of the numerator and denominator polynomials of the associated transfer function. A system is called flat when this difference is equal to the number of the system states, i.e. a flat output exists in principle.

In case of noisy measurements and model imperfections the Kalman Filter is a reasonable alternative.

\section{A.3. Kalman Filtering}

The underlying idea is to extend the system states, $x$, by the system inputs, $u$, according to:

$$
x^{\ddagger}=[x, u]^{\top}
$$

In the field of unknown input reconstruction (Witczak, 2014), the inputs are frequently treated as Gaussian random variables, i.e. $[u]_{i} \sim \mathcal{N}\left({\overline{[u]_{i}}}_{i}, \sigma_{u}^{2}\right)$. The time-discrete version of the corresponding state-space system is given by:

$$
\begin{aligned}
x_{k}^{\ddagger} & =A^{\ddagger} x_{k-1}^{\ddagger}+w_{k-1} \\
y_{k} & =C^{\ddagger} x_{k}^{\ddagger}+v_{k}
\end{aligned}
$$

where $A^{\ddagger}$ and $B^{\ddagger}$ are the adapted dynamic matrix and output matrix. Moreover, $w_{k}$ and $v_{k}$ represent the process noise and the measurement noise (Gelb, 1974), respectively. Within the Kalman Filter framework recent measurements, $y_{k+1}^{D}$, are utilized to reconstruct the extended state vector, $x_{k+1}^{\ddagger,(+)}$, based on a preliminary model-based prediction, $x_{k+1}^{\ddagger},(-)$, as:

$$
x_{k+1}^{\ddagger},(+)=x_{k+1}^{\ddagger,(-)}+K_{k+1}\left(y_{k+1}^{D}-C^{\ddagger} x_{k+1}^{\ddagger}\right)
$$

Details about the Kalman gain, $K_{k+1}$, can be found among others in (Stengel, 1994). In consequence, the unknown system inputs, $u$, are estimated in parallel with the system states, $x$, and can be evaluated subsequently for the purpose of PHM. Recently, the Kalman Filter has been successfully applied to es- timate the track geometry (Naganuma et al., 2013a). Different types of (non)-linear observer strategies might be applicable in a similar way, too. For instance, a Sliding Mode Observer framework has been proposed reconstructing the road profile by sensors mounted on a heavy vehicle (Imine \& Fridman, 2008).

\section{REFERENCES}

Alexanderian, A. (2013). On spectral methods for variance based sensitivity analysis. Probability Surveys.

Bishop, C. M. (2008). Pattern recognition and machine learning. Springer.

Bocciolone, M., Caprioli, A., Cigada, A., \& Collina, A. (2007). A measurement system for quick rail inspection and effective track maintenance strategy. Mechanical Systems and Signal Processing, 21(3), 1242-1254.

Borgonovo, E. (2007). A new uncertainty importance measure. Reliab. Eng. Sys. Safety, 92, 771-784.

Buchholz, J., \& v. Grünhagen, W. (2007). Inversion impossible. GRIN Publishing GmbH.

Caprioli, A., Cigada, A., \& Raveglia, D. (2007). Rail inspection in track maintenance: A benchmark between the wavelet approach and the more conventional fourier analysis. Mechanical Systems and Signal Processing, 21(2), 631-652.

Chiachio, J., Chiachio, M., Saxena, A., Sankararaman, S., Rus, G., \& Goebel, K. (2015). Bayesian model selection and parameter estimation for fatigue damage progression models in composites. International Journal of Fatigue.

Daigle, M., \& Goebel, K. (2010). Improving computational efficiency of prediction in model-based prognsotics using the unscented transformation. Procceedings of the Annual Conference of the Prognostics and Health Management Society.

Daigle, M., \& Sankararaman, S. (2013). Advanced methods for determining prediction uncertainty in model-based prognostics with application to planetary rovers. Annual Conference of the Prognostics and Health Management Society.

Daigle, M., Saxena, A., \& Goebel, K. (2012). An efficient deterministic approach to model-based prediction uncertainty estimation. Annual Conference of the Prognostics and Health Management Society.

Evans, D. H. (1967). An application of numerical integration techniques to statistical tolerancing. Technometrics, 9, 441-456.

Evans, D. H. (1974). Statistical tolerancing: The state of the art. Journal of Quality Technology, 6, 188-195.

Feldmann, U., Kreuzer, E., \& Pinto, F. (2000). Dynamic diagnosis of railway tracks by means of the karhunenloève transformation. Nonlinear Dynamics, 22(2), 183193. 
Fliess, M., Lévine, J., Martin, P., \& Rouchon, P. (1992). On differentially flat non-linear systems. C.R. de l'Académie des Sciences, 315, 619-624.

Fliess, M., Lévine, J., Martin, P., \& Rouchon, P. (1995). Flatness and defect of nonlinear systems: Introductory theory and examples. Int. J. Control, 61, 1327-1361.

Gelb, A. (1974). Applied optimal estimation. The M.I.T. Press.

Gradinariu, T., Turs $\varnothing$-Finnich, K., Juntti-Espling, U., Mangan, C., Thill, L., Gebhardt, M., ... Gardin, D. (2008). Lasting infrastructure cost benchmarking (licb): Summary report december 2008.

Graichen, K., Hagenmeyer, V., \& Zeitz, M. (2005). A new approach for inversion-based feedforward control design of non-linear systems. Trans. Autom. Control, 41, 2033-2041.

Grigoriu, M. (2002). Stochastic calculus: Applications in science and engineering. Birkhäuser.

Imine, H. (2011). Sliding mode based analysis and identification of vehicle dynamics. Springer.

Imine, H., \& Fridman, L. (2008). Road profile estimation in heavy vehicle dynamics simulation. International Journal of Vehicle Design.

International Union of Railways (UIC). (2010). Monitoring track condition to improve asset management: Synthesis report of the uic track condition monitoring working group.

Isukapalli, S. S. (1999). Uncertainty analysis of transporttransformation models (Unpublished doctoral dissertation). Rutgers, The State University of New Jersey.

Julier, S. J., \& Uhlmann, J. K. (1994). A general method for approximating nonlinear transformations of probability distributions (Tech. Rep.). Dept. of Engineering Science, University of Oxford.

Kawasaki, J., \& Youcef-Toumi, K. (2002). Estimation of rail irregularities. In 2002 american control conference (pp. 3650-3660).

Kobayashi, M., Naganuma, Y., Nakagawa, M., \& Okumura, T. (2008). Digital inertial algorithm for recording track geometry on commercial shinkansen trains. In Computers in railways xi (Vol. 103, pp. 683-692).

Kobayashi, T., Naganuma, Y., \& Tsunashima, H. (2013). Condition monitoring of shinkansen tracks based on inverse analysis. Chemical Engineering Transactions, 33, 703-708.

Kulkarni, C. S., Biswas, G., Celaya, J. R., \& Goebel, K. (2013). Physics based degradation models for electronic capacitor prognostics under thermal overstress conditions. International Journal of Prognostics and Health Management.

Lapira, E., Brisset, D., Davari, H., Siegel, D., \& Lee, J. (2012). Wind turbine performance assessment using multi-regime modeling approach. Renewable Energy, $45,86-95$.
Lee, J. S., Choi, S., Kim, S.-S., Park, C., \& Kim, Y. G. (2012). A mixed filtering approach for track condition monitoring using accelerometers on the axle box and bogie. IEEE Transactions on Instrumentation and Measurement, 61(3), 749-758.

Lerner, U. N. (2002). Hybrid bayesian networks for reasoning about complex systems (Unpublished doctoral dissertation). Stanford University.

Lewis, R., \& Richards, A. (1988). A compensated accelerometer for the measurement of railway track crosslevel. IEEE Transactions on Vehicular Technology, 37(3), 174178.

Lewis, R. B., \& Richards, A. N. (1986). A new method for the routine measurement of railhead corrugations. Rail International(2), 37-41.

Maitre, O. P. L., \& Knio, O. M. (2010). Spectral methods for uncertainty quantification. Springer.

Molodova, M., Li, Z., \& Dollevoet, R. (2011). Axle box acceleration: Measurement and simulation for detection of short track defects. Wear, 271(1-2), 349-356.

Molodova, M., Li, Z., Nunez, A., \& Dollevoet, R. (2014). Automatic detection of squats in railway infrastructure. IEEE Transactions on Intelligent Transportation Systems, 15(5), 1980-1990.

Mori, H., Sato, Y., Ohno, H., Tsunashima, H., \& Saito, Y. (2013). Development of compact size onboard device for condition monitoring of railway tracks. Journal of Mechanical Systems for Transportation and Logistics, 6(2), 142-149.

Mori, H., Tsunashima, H., Kojima, T., Matsumoto, A., \& Mizuma, T. (2010). Condition monitoring of railway track using in-service vehicle. Journal of Mechanical Systems for Transportation and Logistics, 3(1), 154165.

Murray-Smith, D. J. (2011). Feedback mehtods for inverse simulation of dynamic models for engineering systems applications. Mathematical and Computer Modelling of Dynamical Systems, 17, 515-541.

Murray-Smith, D. J. (2013). The application of parameter sensitivity analysis methods to inverse simulation models. Mathematical and Computer Modelling of Dynamical Systems, 19, 67-90.

Naganuma, Y., Kobayashi, M., \& Okumura, T. (2010). Inertial measurement processing techniques for track condition monitoring on shinkansen commercial trains. Journal of Mechanical Systems for Transportation and Logistics, 3(1), 315-325.

Naganuma, Y., Kobayashi, T., \& Tsunashima, H. (2013a). Track geometry estimation for car-body motions of railway vehicle. Journal of Mechanical Systems for Transportation and Logistics.

Naganuma, Y., Kobayashi, T., \& Tsunashima, H. (2013b). Track geometry estimation from car-body motions of railway vehicle. Journal of Mechanical Systems for 
Transportation and Logistics, 6(2), 133-141.

Saha, B., Goeble, K., Poll, S., \& Christophersen, J. (2009). Prognostics methods for battery health monitoring using a bayesian framework. IEEE TRANSACTIONS ON INSTRUMENTATION AND MEASUREMENT, 52(2).

Saltelli, A., Ratto, M., Tarantola, S., \& Campolongo, F. (2005). Sensititivity analysis for chemical models. Chemical Reviews, 105, 2811-2828.

Sankararaman, S. (2012). Uncertainty quantification and integration in engineering systems (Unpublished doctoral dissertation). Vanderbilt University.

Sankararaman, S., Daigle, M., Saxena, A., \& Goebel, K. (2013). Analytical algorithms to quantify the uncertainty in remaining useful life prediction. In Aerospace conference.

Sankararaman, S., \& Goebel, K. (2013). Uncertainty quantification in remaining useful life of aerospace components using state space models and inverse form (Tech. Rep.). NASA Ames Research Center; Moffett Field, CA, United States.

Schenkendorf, R. (2014). A general framework for uncertainty propagation based on point estimate methods. In Second european conference of the prognostics and health management society, phme14. Nantes, France.

Schöniger, A., Nowak, W., \& Franssen, H.-J. H. (2012). Parameter estimation by ensemble kalman filters with transformed data: Approach and application to hydraulic tomography. Water Resources Research, 48.

Sira-Ramirez, H., Matamoros-Sanchez, A., \& Goodall, R. M. (2011). Flatness based control of a suspension system: A gpi observer approach. In 18th ifac world congress.

Sobol', I. M. (1993). Sensitivity analysis for nonlinear mathematical models. Mathematical Modeling and Computational Experiment, 1, 407-414.

Sobol', I., Tarantola, S., Gatelli, D., Kucherenko, S., \& Mauntz, W. (2007). Estimating the approximation error when fixing unessential factors in global sensitivity analysis. Reliability Engineering \& System Safety, 92(7), 957 - 960.

Stengel, R. F. (1994). Optimal control and estimation. Dover Publications.

Sudret, B. (2007). Uncertainty propagation and sensitivity analysis in mechanical models. Universite BLAISE PASCAL - Clermont II.

Sunaga, Y., Sano, I., \& Ide, T. (1997). A method to control the short wave track irregularities utilizing axlebox acceleration. Railway Technical Research Institute, Quarterly Reports, 38(4), 176-181.

Thomson, D., \& Bradley, R. (2006). Inverse simulation as a tool for fight dynamics research - principles and applications. Progress in Aerospace Sciences, 42, 174210.

Tyler, G. W. (1953). Numerical integration of functions of several variables. Canadian Jn. Math., 5, 393-412.
Ward, C., Weston, P., Stewart, E. J. C., Li, H., Goodall, R., Roberts, C., ... Dixon, R. (2011). Condition monitoring opportunities using vehicle-based sensors. Proceedings of the Institution of Mechanical Engineers, Part F: Journal of Rail and Rapid Transit, 225(2), 202-218.

Weston, P., Ling, C., Goodman, C., Roberts, C., Li, P., \& Goodall, R. (2007). Monitoring lateral track irregularity from in-service railway vehicles. Proceedings of the Institution of Mechanical Engineers, Part F: Journal of Rail and Rapid Transit, 221(1), 89-100.

Weston, P., Ling, C., Roberts, C., Goodman, C., Li, P., \& Goodall, R. (2007). Monitoring vertical track irregularity from in-service railway vehicles. Proceedings of the Institution of Mechanical Engineers, Part F: Journal of Rail and Rapid Transit, 221(1), 75-88.

Williard, N., He, W., Osterman, M., \& Pecht, M. (2013). Comperative analysis of features for determining state of health in lithium-ion batteries. International Journal of Prognostics and Health Management.

Witczak, M. (2014). Fault diagnosis and fault-tolerant control strategies for non-linear system: Analytical and soft computing approaches. Springer.

Zhang, X., \& Pisu, P. (2014). Prognostic-oriented fuel cell catalyst aging modeling and its application to healthmonitoring and prognostics of a pem fuel cell. International Journal of Prognostics and Health Management, 5.

\section{BIOGRAPHIES}

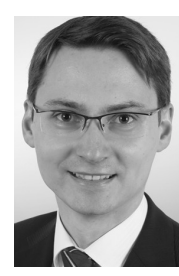

René Schenkendorf René received his Dipl.-Ing. and Dr.-Ing. in Engineering Cybernetics from the Otto-von-GuerickeUniversity Magdeburg, Germany in 2007 and 2014, respectively. From 2007 until 2012 he had been a Ph.D. student at the Max Planck Institute for Dynamics of Complex Technical Systems in Magdeburg, Germany. Since 2013 he is with the German Aerospace Center at the Institute of Transportation Systems in Brunswick, Germany. His current research interests include data analysis, statistical process control, uncertainty propagation, and modeling in PHM.

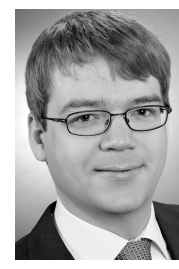

Jörn C. Groos Jörn received is diploma in geophysics from the University of Karlsruhe, Germany, in 2007 and his Dr. rer. nat. from the faculty of physics of the Karlsruhe Institute of Technology in 2010. Since 2014 he is with the German Aerospace Center at the Institute of Transportation Systems in Brunswick, Germany. His current research interests include data acquisition, data analysis, and pattern recognition for condition monitoring of railway tracks. 